\title{
Alcohol and the regulation of energy balance: overnight effects on diet-induced thermogenesis and fuel storage
}

\author{
BY P. R. MURGATROYD, M. L. H. M. VAN DE VEN, G. R. GOLDBERG \\ AND A. M. PRENTICE \\ MRC Dunn Clinical Nutrition Centre, Hills Road, Cambridge CB2 2DH
}

(Received 23 December 1994-Accepted 3 May 1995)

\begin{abstract}
The effect of alcohol on overnight energy expenditure and substrate disposal was studied in eleven subjects (five men, six women) using whole-body indirect calorimetry for $15.5 \mathrm{~h}$ after test meals. Three test meals were studied in random order with at least $48 \mathrm{~h}$ between treatments: control, $50 \%$ of maintenance energy needs provided as 14, 40 and $46 \%$ energy from protein, fat and carbohydrate respectively; alcohol addition, control plus $\mathbf{2 3} \%$ energy as alcohol; alcohol substitution, control with alcohol replacing $23 \%$ of carbohydrate energy. ANOVA revealed no significant sex effects. Alcoholinduced thermogenesis dissipated only 15 (SD 14) \% of the alcohol energy. Alcohol addition had no significant effect on protein or carbohydrate oxidation but fat oxidation was suppressed $(P<0.0005)$ to an extent equivalent to storing 74 (SD 51) \% of the alcohol energy as fat. Alcohol substitution reduced carbohydrate oxidation $(P<0.009)$ to an equivalent of 42 (SD 41$) \%$ and also spared fat $(P<0.005)$ to an equivalent of 59 (SD 37) \% of the alcohol energy. It is concluded that alcohol has no special thermogenic capacity, and that its energy can be accounted for in a similar way to carbohydrate.
\end{abstract}

Alcohol: Fat storage: Whole-body calorimetry

Alcohol consumption in most Western countries increased substantially between 1950 and 1975. Only in recent years has consumption stabilized, at a level of intake almost twice that in 1950 (Sulkunen, 1976; Smart, 1989). The negative health effects of excessive alcohol intake are well known. However, the metabolic fate of the energy ingested as alcohol remains a matter of controversy. The body has no capacity to store alcohol and so might be expected to oxidize it in preference to other energy substrates, sparing them from oxidation and leading ultimately to fat storage. This supposition has recently been brought into question by the epidemiological studies of 48000 men and 89000 women by Colditz et al. (1991). In these studies it appeared that alcohol was consumed in addition to other foods, yet the alcohol-drinking men were no fatter than those who did not drink and the women showed a decrease in adiposity as alcohol intake increased. These observations imply total dissipation of the alcohol energy; in fact more than total dissipation in women. The Colditz et al. (1991) paper has suggested a paradox in the metabolic consequences of alcohol ingestion and has led to a comprehensive re-appraisal of our understanding of alcohol metabolism (Leibel et al. 1993).

There is no immediately obvious mechanism for dissipating energy ingested as ethanol. Lieber et al. (1988) have proposed that uncoupled oxidation by the microsomal ethanoloxidizing system (MEOS) may dissipate energy by partially replacing the fully coupled oxidation by alcohol dehydrogenase $(E C$ 1.1.1.1; $\mathrm{ADH})$ in habitual moderate or heavy drinkers. However, a substantial proportion of the ethanol would still be oxidized via the $\mathrm{ADH}$ route and the acetaldehyde resulting from either route would retain most of the ethanol's initial energy. Thus, the potential for ethanol-induced thermogenesis by the 
MEOS route is small. An alternative proposal by Lands \& Zakhari (1991) is that a futile cycle links ethanol and acetaldehyde, dissipating 6 ATP equivalents at each turn. After only two or three cycles the energy of the ethanol feeding the cycle would be lost. If such dissipative mechanisms as these were operating then the energy consumed by them could account for the findings of Colditz et al. (1991) but would be easily measurable as alcoholinduced thermogenesis. As long ago as 1940, Carpenter (1940) had concluded that less than $10 \%$ of the energy of alcohol is dissipated as post-ingestive thermogenesis. Weststrate et al. (1990) have recently reported a similar value: about $10.2 \%$ of alcohol energy dissipated. The Carpenter (1940) and Weststrate et al. (1990) studies, however, lasted for 1.5-4 $\mathrm{h}$ after the alcohol ingestion and may have failed to reveal delayed thermogenesis suggested in the studies of Rosenberg \& Durnin (1978). Weststrate et al. (1990) indicate that the thermogenesis in their study was not complete at the end of $4 \mathrm{~h}$.

If alcohol is oxidized without total dissipation of its energy this must have implications for the management of other energy substrates. Shelmet et al. (1988) have shown that infused ethanol inhibits the oxidation of fat and, to a lesser extent, protein and carbohydrate, during a $4 \mathrm{~h}$ period after ethanol administration. Suter et al. (1992) in $24 \mathrm{~h}$ manipulations have reported suppression of fat oxidation by both the addition of ethanol ( $25 \%$ by energy) to a normal diet and by the substitution of ethanol for $25 \%$ of the energy, taken equally from fat and carbohydrate. They found no significant impact of the ethanol on protein or carbohydrate oxidation (Suter et al. 1992). Sonko et al. (1994) in our laboratory have studied the effects of alcohol consumed with lunch. Lunches were served without alcohol, with additional alcohol and with alcohol substituted for carbohydrate. The effects of the lunch were followed for $20.5 \mathrm{~h}$, with an alcohol-free evening meal served after $6 \mathrm{~h}$ to enable any re-establishment of substrate balance to be disclosed. Alcohol addition was shown to lead to a residual positive fat balance at the end of the $20.5 \mathrm{~h}$ period. However, substitution of carbohydrate by alcohol, whilst initially leading to fat storage, had no significant residual effect by the end of the study.

The present paper presents the findings of a study designed to complement and extend those reviewed previously. Its aim was to examine both the thermogenic effects of the alcohol and its influence on the selection of other energy substrates both during and after the period of alcohol oxidation. Alcohol was given with a normal meal served early in the evening and its metabolic consequences followed throughout the subsequent night, a total outcome period of $15.5 \mathrm{~h}$. Alcohol was given in addition to the meal on one occasion and as a partial substitute for carbohydrate alone on another, so that we could divorce the fat outcome effects reported by others from fat intake manipulations. Finally, both male and female groups were studied in the light of reports that reduced gastric ADH activity in women resulted in proportionally higher blood alcohol responses than those seen in men (Frezza et al. 1990), and in view of the sex differences in the response of body composition to habitual alcohol intake disclosed by the Colditz et al. (1991) study.

\section{METHODS}

\section{Subjects and experimental protocol}

Eleven healthy volunteers were recruited from employees at the Dunn Clinical Nutrition Centre and from volunteers known to us from previous studies. Their physical characteristics are presented in Table 1. Five subjects were male and six were female; the additional female subject was recruited as two female subjects failed to complete the whole study. Informed written consent was obtained from each volunteer, and their fitness to participate confirmed by a medical consultation. The subjects were not habitually heavy 
Table 1. Physical characteristics of the subjects

\begin{tabular}{|c|c|c|c|c|c|}
\hline & $\begin{array}{c}\text { Subject } \\
\text { no. }\end{array}$ & $\begin{array}{c}\text { Age } \\
\text { (years) }\end{array}$ & $\begin{array}{l}\mathrm{Wt} \\
(\mathrm{kg})\end{array}$ & $\begin{array}{l}\text { Height } \\
\text { (m) }\end{array}$ & $\underset{\left(\mathrm{kg} / \mathrm{m}^{2}\right)}{\mathrm{BMI}}$ \\
\hline Females & $\begin{array}{l}\text { I } \\
2 \\
3 \\
4 \\
\\
\\
\text { Mean } \\
6 \\
\text { SD }\end{array}$ & $\begin{array}{c}22 \\
24 \\
33 \\
22 \\
28 \\
44 \\
28 \cdot 8 \\
8 \cdot 5\end{array}$ & $\begin{array}{l}60 \\
52 \\
65 \\
70 \\
60 \\
61 \\
61 \cdot 3 \\
6 \cdot 0\end{array}$ & $\begin{array}{l}1.54 \\
1.55 \\
1.60 \\
1.78 \\
1.75 \\
1.64 \\
1.64 \\
0.10\end{array}$ & $\begin{array}{r}25.3 \\
21.6 \\
25 \cdot 4 \\
22 \cdot 0 \\
20.0 \\
23.0 \\
22.8 \\
1.9\end{array}$ \\
\hline Males & $\begin{array}{r}7 \\
8 \\
9 \\
10 \\
11 \\
11 \\
\text { Mean } \\
\text { SD }\end{array}$ & $\begin{array}{l}21 \\
37 \\
49 \\
29 \\
26 \\
32 \cdot 2 \\
10 \cdot 9\end{array}$ & $\begin{array}{l}69 \\
72 \\
70 \\
74 \\
82 \\
73 \cdot 6 \\
5 \cdot 2\end{array}$ & $\begin{array}{l}1.77 \\
1.73 \\
1.79 \\
1.79 \\
1.80 \\
1.78 \\
0.03\end{array}$ & $\begin{array}{r}22 \cdot 0 \\
23 \cdot 9 \\
22 \cdot 0 \\
23 \cdot 0 \\
25 \cdot 1 \\
23 \cdot 2 \\
1 \cdot 3\end{array}$ \\
\hline
\end{tabular}

drinkers and were asked to forgo alcohol for $48 \mathrm{~h}$ before the study. The study had the approval of the Dunn Nutrition Unit's Ethical Committee.

Each subject was studied on three occasions, characterized by different randomlyselected test meals, and acted as his or her own control. At least $2 \mathrm{~d}$ elapsed between study periods. On each visit the subject reported to the Centre at 09.00 hours and remained within the Centre until the end of each experiment. On arrival breakfast was provided, followed at 12.30 hours by lunch. At 16.00 hours the subject entered the calorimeter. Whilst in the calorimeter the subject could watch TV, read, write or listen to the radio, but no unnecessary physical activity was allowed. Just before 17.30 hours the subject was asked to pass urine. Thereafter, the subject could pass urine whenever needed. The urine collections were pooled from 17.30 hours to midnight and from midnight to 09.00 hours. Total urine volume was recorded and duplicate samples were analysed to determine $\mathrm{N}$ excretion.

Dinner, which was the test meal of the study, was served at 17.30 hours, and the subject was asked to eat this within $0.5 \mathrm{~h}$. At 23.30 hours the subject prepared for bed before 'lights out' at 24.00 hours. BMR was measured between 08.00 and 09.00 hours the next morning.

\section{Meals}

The energy content of the control diet was matched, to the nearest $1 \mathrm{MJ}$, to each subject's predicted daily maintenance energy expenditure (MEE). The MEE in the present study was 1.4 times predicted BMR (Schofield et al. 1985). Mean MEE for men was 10.4 (SD 0.55) MJ and for women was 8.2 (SD 0.75) MJ.

Energy intake was divided between meals to provide $25 \%$ MEE for each of breakfast and lunch. The composition of breakfast and lunch was typical of a British diet, i.e. $46 \%$ of energy as carbohydrate, $40 \%$ fat and $14 \%$ protein. Dinner was manipulated as follows: control meal (CON), without alcohol; this had the same composition as breakfast and lunch and provided $50 \%$ MEE, thus keeping the subjects close to energy balance over the study day; alcohol substitution (SUB), in which $50 \%$ of the energy from the carbohydrate of the control meal $(23 \%$ of the energy of the meal) was substituted with energy from alcohol. This meal also provided $50 \%$ MEE and aimed to maintain energy balance; alcohol 
addition (ADD), was equivalent to the control meal with $23 \%$ of the energy added as alcohol. This meal thus provided $61 \cdot 5 \% \mathrm{MEE}$.

Diets of $10 \mathrm{MJ}$ were formulated to meet the criteria described and from these diets covering the range 7-11 MJ were calculated. Breakfast consisted of orange juice, scrambled egg, bread, margarine and jam. Lunch consisted of cheese and salad with apple juice, bread and margarine. Dinner consisted of beef (or cheese for vegetarians) with vegetables and rice. The alcohol was in the form of red wine ( $12 \%$ alcohol by volume; Bergerac Rouge, Chais Réunis Négociants à Blanquefort, Gironde, France). The manipulation of the $10 \mathrm{MJ}$ diet required $38.75 \mathrm{~g}$ ethanol. From arrival at the Centre until the end of the test period subjects were allowed black decaffeinated coffee or tea ad lib.

\section{Calorimetry}

Energy expenditure and substrate oxidation rates were measured by whole-body indirect calorimetry. The present study employed the two small calorimeters at the former Dunn Clinical Nutrition Centre. The calorimeters were small rooms of $11 \mathrm{~m}^{3}$ volume. Each was furnished with a comfortable chair, folding bed, table, radio and television. A telephone and intercom provided communication with the outside world. Food was passed into the calorimeter through an air-lock hatch. The calorimeter temperature was maintained at $24 \pm 0 \cdot 5^{\circ}$.

The air within the calorimeter was recirculated at a rate of $20 \mathrm{~m}^{3} / \mathrm{min}$ to mix the subject's expired air with the room air. The calorimeter was ventilated with fresh air which was pumped into the chamber at $200 \mathrm{l} / \mathrm{min}$. Samples of ventilating air and mixed calorimeter air were analysed for $\mathrm{O}_{2}$ using a Servomex OA184 paramagnetic $\mathrm{O}_{2}$ analyser and for $\mathrm{CO}_{2}$ using a Servomex $12 \mathrm{X} 1$ single-beam infra red $\mathrm{CO}_{2}$ analysis module (Servomex, Crowborough, Sussex). Data from the analysers were digitized using a Solartron type 3510 integrated measurement system (Solartron, Farnborough, Hants) and passed to a Hewlett Packard measurement co-processor (Hewlett Packard, Palo Alto, California, USA), which adds powerful data acquisition and control features to a standard personal computer. The measurement co-processor controlled the sampling of gases and calculated respiratory gas exchanges using the procedures described by Brown et al. (1984). The accuracy of gasexchange measurements was verified by infusing a mixture of $\mathrm{CO}_{2}(200 \mathrm{ml} / \mathrm{l})$ with $\mathrm{N}_{2}$ $(800 \mathrm{ml} / \mathrm{l})$ into the calorimeter at a measured rate (Murgatroyd et al. 1987).

\section{Alcohol measurement}

Plasma alcohol was estimated from breath alcohol measurements made using the Lion Alcometer AE-D3 (Lion Laboratories, Barry, South Glamorgan). The instrument was calibrated using a Nalco alcohol vapour standard supplied by the manufacturers.

Alcohol oxidized, $A(\mathrm{~g})$ was calculated as:

$$
A=\Delta A_{p} \times \mathrm{BW} \times k_{a} \times 1.06,
$$

where $\Delta A_{p}$ is the change in plasma alcohol concentration $(\mathrm{g} / 1), \mathrm{BW}$ is body weight $(\mathrm{kg}), k_{a}$ is the alcohol distribution coefficient ( 0.69 for men, 0.57 for women; Carpenter, 1940) and the factor 1.06 corrects for the difference between the density of plasma and water (Shelmet et al. 1988).

\section{Substrate oxidation calculations}

The rate of oxidation of protein was calculated from estimation of urinary $\mathrm{N}$ excretion by Kjeldahl analysis of urine samples. Fat and carbohydrate oxidations were calculated from $\mathrm{O}_{2}$ consumption and $\mathrm{CO}_{2}$ production corrected for the exchanges of these gases associated with protein and alcohol oxidation (Murgatroyd et al. 1993). The constants used in these 
Table 2. Constants used in the calculation of substrate oxidation rates

\begin{tabular}{llcl}
\hline Substrate & $\begin{array}{c}\mathrm{RQ} \text { of } \\
\text { oxidation }\end{array}$ & $\begin{array}{c}\mathrm{O}_{2} \text { consumption } \\
(\mathrm{l} / \mathrm{g})\end{array}$ & $\begin{array}{c}\text { Heat production } \\
(\mathrm{kJ} / \mathrm{g})\end{array}$ \\
\hline Fat & 0.71 & 2.01 & 39.33 \\
Carbohydrate & 1.0 & 0.746 & 15.76 \\
Protein & 0.835 & 0.952 & 18.56 \\
Alcohol & 0.667 & 1.461 & 29.68 \\
\hline
\end{tabular}

calculations are given in Table 2 and are from the work of Livesey \& Elia (1988). The calculations assumed that measurements were made under equilibrium conditions, e.g. that alcohol which has disappeared from the blood was completely oxidized, and that the blood urea and lactate levels remained constant.

\section{Statistical analysis}

Data for total energy expenditure and for protein, carbohydrate and fat oxidation were analysed by ANOVA and the Scheffé post hoc test for differences between CON $v$. SUB and CON $v$. ADD diets and to disclose any differences between the responses of male and female groups. Significance has been assumed for $P<0.05$.

\section{RESULTS}

\section{Blood alcohol}

Fig. 1 shows the alcohol disappearance curves following the alcohol-added meal. There was a tendency for the men to show a lower blood alcohol level than the women during the first $3 \mathrm{~h}$ after the meal, but the two disappearance curves were not significantly different. Alcohol disappearance on the SUB protocol followed similar curves to those of Fig. 1 and on both SUB and ADD protocols blood alcohol levels had returned to the control level by the end of the $6.5 \mathrm{~h}$ period between the test dinner and bedtime. Of the alcohol given with the test meals, 72 (SD 12) \% was accounted for by disappearance of blood alcohol. This was not significantly different between men and women. To avoid errors from buccal alcohol residues, the breath alcohol measurements, from which this value was estimated, did not commence until $50 \mathrm{~min}$ after the start of the meal. Extrapolation of the disappearance curve back to the start of the meal accounts for almost all the underestimate of alcohol oxidized, although small contributions, perhaps $2 \%$, would have arisen from alcohol loss in breath and urine (Shelmet et al. 1988).

\section{Energy and substrate oxidation data}

Data for energy expenditure and substrate oxidation have been analysed in two periods; 17.30 hours until bedtime at 24.00 hours $(0-6.5 \mathrm{~h})$ and 24.00 hours to the end of the study at 09.00 hours $(6 \cdot 5-15 \cdot 5 \mathrm{~h})$. The analysis intervals correspond to the waking period, while alcohol was disappearing from the blood, and the sleeping period, after blood alcohol had returned to control levels. The data from these periods, together with the data for the whole duration of the study $(0-15.5 \mathrm{~h})$, are summarized in Table 3 for the men and Table 4 for the women.

All five men completed the three measurements. Of the six women studied, subject 2 only completed CON and ADD measurements and subject 3 only completed CON and SUB. Statistical analyses were performed on the pooled data for male and female subjects and 


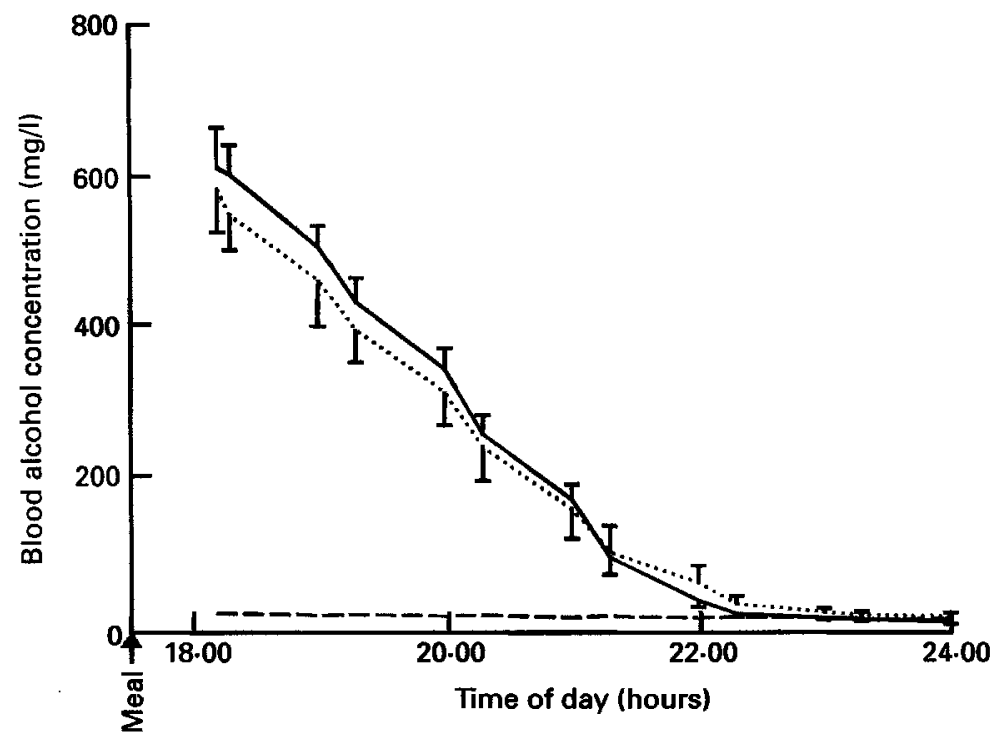

Fig. 1. Changes in blood alcohol concentration with time after a test meal in which $23 \%$ of the energy was added as alcohol for men $(\cdots)$ and women $(-)$ and baseline levels $(--)$ from pooled control measurements. The control meal provided $50 \%$ daily predicted maintenance energy expenditure (MEE), while the test meal with alcohol provided $61.5 \%$ daily predicted MEE (for details of protocols, see pp. 34-36). Values are means with their standard errors represented by vertical bars.

Table 3. Mean substrate oxidations $(k J)$ for male subjects receiving a test meal with (SUB, $A D D)$ or without (CON) alcohol

(Mean values and standard deviations)

\begin{tabular}{|c|c|c|c|c|c|c|c|}
\hline $\begin{array}{c}\text { Period after } \\
\text { meal (h) }\end{array}$ & \multicolumn{2}{|c|}{ Meal* } & Alcohol & Protein & $\mathrm{CHO}$ & Fat & Energy \\
\hline \multirow[t]{5}{*}{$0-6.5$} & CON: & $\begin{array}{l}\text { Mean } \\
\text { SD }\end{array}$ & 0 & $\begin{array}{l}522 \\
175\end{array}$ & $\begin{array}{l}997 \\
142\end{array}$ & $\begin{array}{r}1336 \\
227\end{array}$ & $\begin{array}{r}2855 \\
151\end{array}$ \\
\hline & SUB: & Mean & 1196 & 531 & 748 & 400 & 2877 \\
\hline & & SD & 63 & 112 & 80 & 112 & 197 \\
\hline & ADD: & Mean & 1196 & 463 & 890 & 495 & 3045 \\
\hline & & SD & 63 & 112 & 175 & 227 & 256 \\
\hline \multirow[t]{6}{*}{$6 \cdot 5-15 \cdot 5$} & CON: & Mean & 0 & 449 & 818 & 1299 & 2566 \\
\hline & & SD & & 99 & 185 & 209 & 195 \\
\hline & SUB: & Mean & 0 & 506 & 787 & 1366 & 2659 \\
\hline & & SD & & 141 & 28 & 175 & 189 \\
\hline & ADD: & Mean & 0 & 422 & 999 & 1234 & 2655 \\
\hline & & SD & & 107 & 170 & 365 & 272 \\
\hline \multirow[t]{6}{*}{$0-15 \cdot 5$} & CON: & Mean & 0 & 970 & 1815 & 2635 & 5420 \\
\hline & & SD & & 260 & 307 & 396 & 297 \\
\hline & SUB: & Mean & 1196 & 1038 & 1537 & 1766 & 5536 \\
\hline & & SD & 63 & 212 & 83 & 207 & 380 \\
\hline & ADD: & Mean & 1196 & 885 & 1889 & 1730 & 5700 \\
\hline & & SD & 63 & 199 & 293 & 536 & 521 \\
\hline
\end{tabular}

$\mathrm{CON}$, the control meal provided $50 \%$ daily predicted maintenance energy expenditure (MEE); SUB, in the test meal $50 \%$ of the energy from carbohydrate of the control meal ( $23 \%$ of the energy of the meal) was replaced by alcohol, the meal providing $50 \%$ daily predicted MEE; ADD, in the test meal $23 \%$ of the energy of the control meal was added as alcohol, the meal supplying $61.5 \%$ daily predicted MEE; CHO, carbohydrate.

* For details of protocols, see pp. 34-37. 
Table 4. Mean substrate oxidations $(k J)$ for female subjects receiving a test meal with $(S U B, A D D)$ or without $(C O N)$ alcohol

(Mean values and standard deviations)

\begin{tabular}{|c|c|c|c|c|c|c|c|}
\hline $\begin{array}{l}\text { Period after } \\
\text { meal (h) }\end{array}$ & $\mathrm{Me}$ & & Alcohol & Protein & $\mathrm{CHO}$ & Fat & Energy \\
\hline \multirow[t]{6}{*}{$0-6.5$} & CON: & Mean & 0 & 324 & 877 & 1021 & 2223 \\
\hline & & SD & & 58 & 144 & 181 & 243 \\
\hline & SUB: & Mean & 965 & 366 & 571 & 423 & 2325 \\
\hline & & SD & 64 & 46 & 266 & 105 & 259 \\
\hline & ADD: & Mean & 965 & 350 & 708 & 344 & 2367 \\
\hline & & SD & 64 & 69 & 114 & 73 & 266 \\
\hline \multirow[t]{6}{*}{$6 \cdot 5-15 \cdot 5$} & CON: & Mean & 0 & 401 & 796 & 928 & 2125 \\
\hline & & SD & & 105 & 291 & 266 & 222 \\
\hline & SUB: & Mean & 0 & 369 & 548 & 1219 & 2136 \\
\hline & & SD & & 57 & 428 & 254 & 198 \\
\hline & ADD: & Mean & 0 & 364 & 916 & 834 & 2114 \\
\hline & & SD & & 129 & 198 & 147 & 235 \\
\hline \multirow[t]{6}{*}{$0-15.5$} & CON: & Mean & 0 & 725 & 1673 & 1949 & 4347 \\
\hline & & SD & & 136 & 403 & 433 & 456 \\
\hline & SUB: & Mean & 965 & 734 & 1119 & 1642 & 4461 \\
\hline & & SD & 64 & 102 & 689 & 347 & 452 \\
\hline & ADD: & Mean & 965 & 714 & 1624 & 1178 & 4481 \\
\hline & & SD & 64 & 191 & 295 & 204 & 484 \\
\hline
\end{tabular}

$\mathrm{CON}$, the control meal provided $50 \%$ daily predicted maintenance energy expenditure (MEE); SUB, in the test meal $50 \%$ of the energy from carbohydrate of the control meal ( $23 \%$ of the energy of the meal) was replaced by alcohol, the meal providing $50 \%$ daily predicted MEE; ADD, in the test meal $23 \%$ of the energy of the control meal was added as alcohol, the meal supplying $61.5 \%$ daily predicted MEE; CHO, carbohydrate.

* For details of protocols, see pp. 34-37.

were tested for differences in male-female response, however none was found. The impact of the manipulations is illustrated by Figs. $2-5$ which have been generated from the pooled data; these show the changes in energy expenditure, protein, carbohydrate and fat oxidation on the SUB and ADD protocols relative to the CON protocol. The $P$-values of statistically significant outcomes are given in the text (see p. 39-42).

\section{Energy expenditure}

Energy expenditure was significantly increased by 162 (SD 173) $\mathrm{kJ}$, during $6.5 \mathrm{~h}$ after the ADD meal when compared with the CON protocol $(P<0.02)$. This increase was, on average, equivalent to $16 \%$ of the energy added by the alcohol for the males $(190 \mathrm{~kJ})$ and $14 \%$ for the females $(144 \mathrm{~kJ})$. The energy expenditure over the full $15.5 \mathrm{~h}$ of the study was greater by 191 (SD 279) $\mathrm{kJ}$ than that on the CON protocol, equivalent to $17.6 \%$ of the energy given as alcohol, though this effect fell just short of significance $(P=0.06)$.

There were no significant differences in energy expenditure between the CON and SUB protocols during any of the analysis intervals (Fig. 2).

\section{Substrate oxidations}

Protein oxidation was not influenced by either the addition or substitution of alcohol during any of the time intervals analysed (Fig. 3).

Carbohydrate oxidation was significantly suppressed during the $6.5 \mathrm{~h}$ after the SUB meal, in which alcohol was substituted for half the carbohydrate (290 (SD 177) kJ, equivalent to $27 \%$ of alcohol energy; $P<0.004$; Fig. 4). Overnight carbohydrate oxidation 


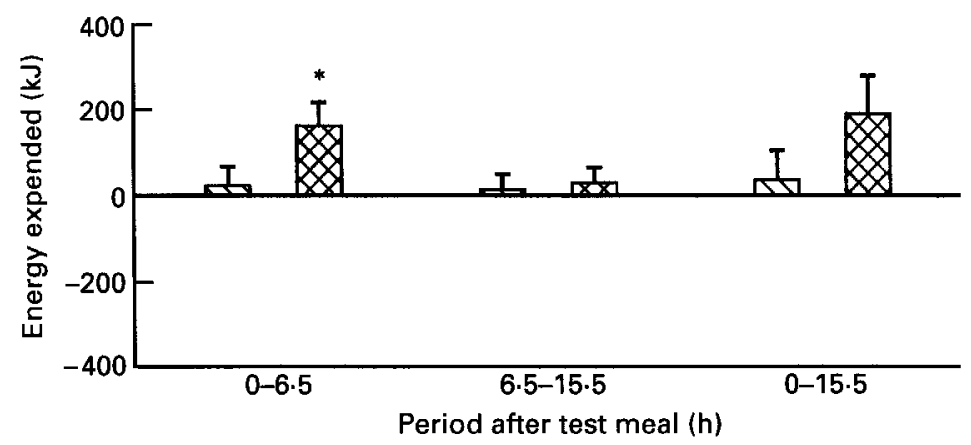

Fig. 2. The effect of alcohol in a test meal on energy expenditure relative to that after a control meal in men and women. The control protocol provided a test meal which supplied $50 \%$ daily predicted maintenance energy expenditure (MEE), in the alcohol substitution ( $\$$ ) protocol $50 \%$ of the energy from carbohydrate of the control meal was replaced by alcohol and the test meal provided $50 \%$ daily predicted MEE, whereas in the alcohol addition (B) protocol $23 \%$ of the energy in the control meal was added as alcohol, the test meal providing $61.5 \%$ daily predicted MEE (for details of protocols, see pp. 34-36). Values are means with their standard errors represented by vertical bars. * Mean values were significantly different from control values.

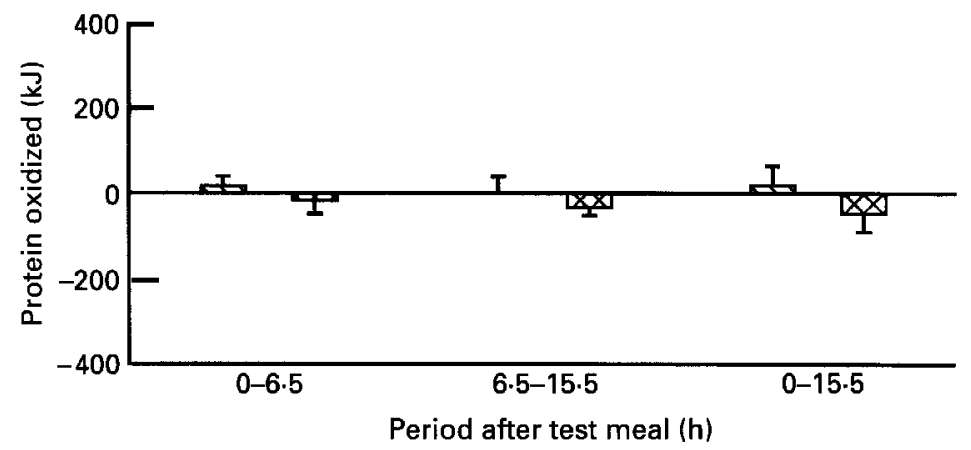

Fig. 3. The effect of alcohol in a test meal on protein oxidation relative to that after a control meal in men and women. The control protocol provided a test meal which supplied $50 \%$ daily predicted maintenance energy expenditure (MEE), in the alcohol substitution $(\$)$ protocol $50 \%$ of the energy from carbohydrate of the control meal was replaced by alcohol and the test meal provided $50 \%$ daily predicted $\mathrm{MEE}$, whereas in the alcohol addition (ख) protocol $23 \%$ of the energy in the control meal was added as alcohol, the test meal providing $61.5 \%$ daily predicted MEE (for details of protocols, see pp. 34-37). Values are means with their standard errors represented by vertical bars.

remained slightly suppressed; consequently over the full $15.5 \mathrm{~h}$ of the study carbohydrate oxidation was significantly suppressed by 449 (SD 432) $\mathrm{kJ}, 42 \%$ of the alcohol-substituted energy $(P<0 \cdot 009)$.

In the $6.5 \mathrm{~h}$ following the ADD meal carbohydrate oxidation was also significantly suppressed although to a lesser degree than after the SUB meal (161 (SD 216) kJ, 15\% of alcohol energy; $P<0.03$ ). There was no significant effect on carbohydrate oxidation over the whole $15 \cdot 5 \mathrm{~h}$ of the ADD protocol.

Fat oxidation showed highly significant suppression relative to $\mathrm{CON}$ during the first $6.5 \mathrm{~h}$ after both the SUB and ADD meals, as can be seen in Fig. $5(P<0.001)$. During the subsequent overnight period fat oxidation was restored, and even slightly elevated after the SUB meal in compensation for the restricted carbohydrate intake. Over all, however, the fat oxidation was lower in every subject following the meals which included alcohol. Suppressed fat oxidation was a significant finding on both the SUB $(P<0.005)$ and ADD 


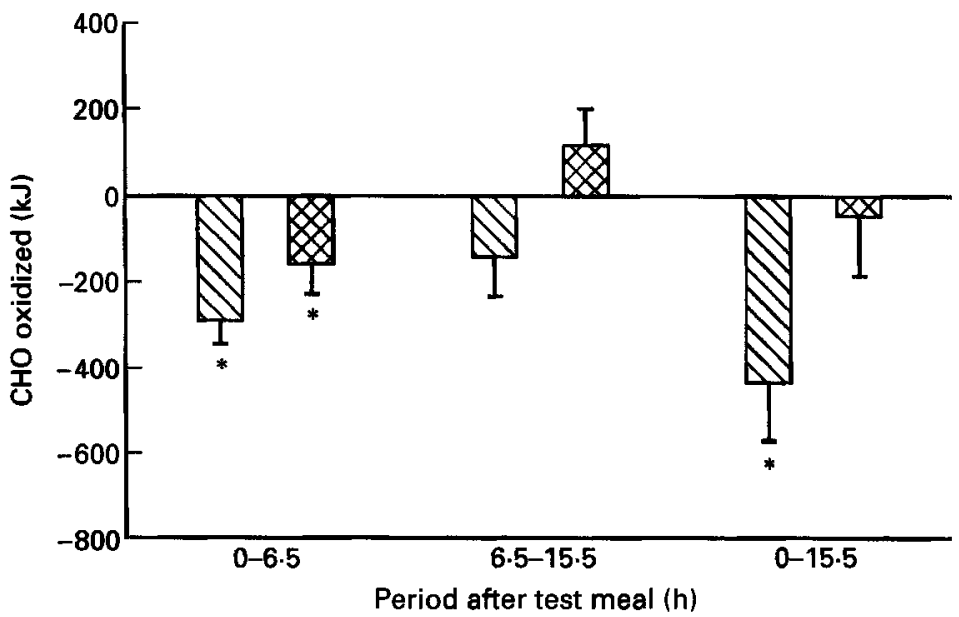

Fig. 4. The effect of alcohol in a test meal on carbohydrate (CHO) oxidation relative to that after a control meal in men and women. The control protocol provided a test meal which supplied $50 \%$ daily predicted maintenance energy expenditure (MEE), in the alcohol substitution ( $\mathrm{\Xi}$ ) protocol $50 \%$ of the energy from carbohydrate of the control meal was replaced by alcohol and the test meal provided $50 \%$ daily predicted MEE, whereas in the alcohol addition (因) protocol $23 \%$ of the energy in the control meal was added as alcohol, the test meal providing $61.5 \%$ daily predicted MEE (for details of protocols, see pp. 34-37). Values are means with their standard errors represented by vertical bars. * Mean values were significantly different from control values.

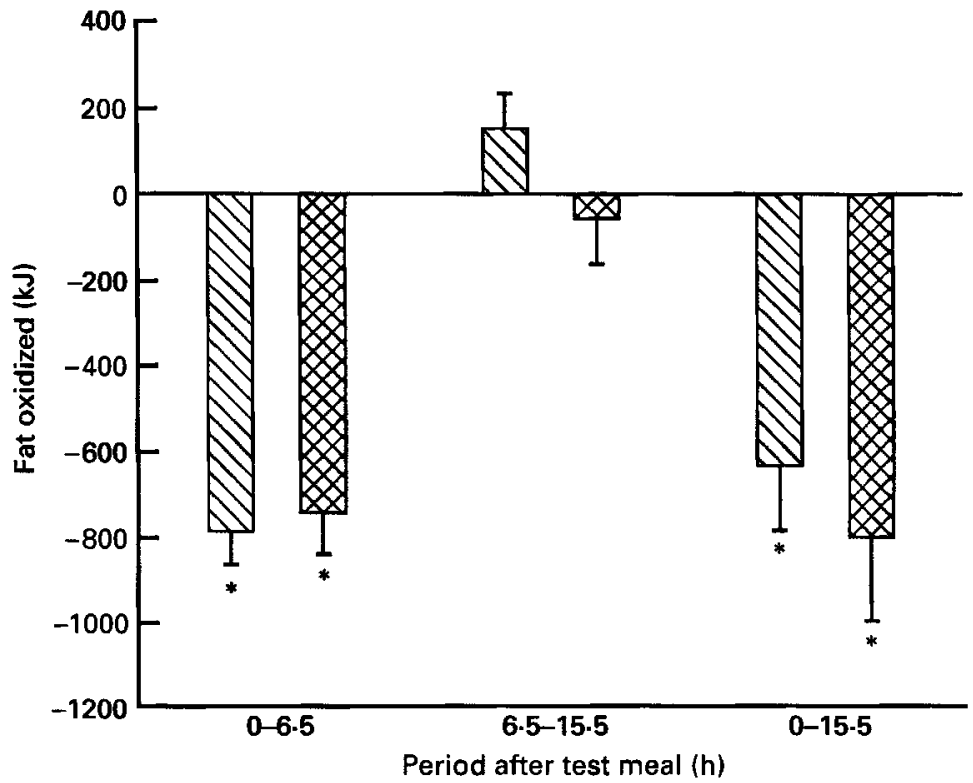

Fig. 5. The effect of alcohol in a test meal on fat oxidation relative to that after a control meal in men and women. The control protocol provided a test meal which supplied $50 \%$ daily predicted maintenance energy expenditure (MEE), in the alcohol substitution ( $\mathbb{Q}$ ) protocol $50 \%$ of the energy from carbohydrate of the control meal was replaced by alcohol and the test meal provided $50 \%$ daily predicted MEE, whereas in the alcohol addition ( $B$ ) protocol $23 \%$ of the energy in the control meal was added as alcohol, the test meal providing $61.5 \%$ daily predicted MEE (for details of protocols, see pp. 34-37). Values are means with their standard errors represented by vertical bars. * Mean values were significantly different from control values. 
$(P<0.0005)$ protocols over the full $15.5 \mathrm{~h}$ duration of the study. Fat oxidation was suppressed by 633 (SD 472) kJ, $59 \%$ of the alcohol energy given, on the SUB protocol and by 796 (SD 621) kJ, $74 \%$ of the alcohol energy, on the ADD protocol.

\section{DISCUSSION}

\section{Blood alcohol measurements}

Alcohol disappeared from the blood in a superficially linear manner down to a concentration of $100 \mathrm{mg} / \mathrm{l}$. This is in accordance with previous observations (Badawy, 1978). During the $6.5 \mathrm{~h}$ period between the meal and sleep the breath alcohol returned to a level indistinguishable from that of the control measurements, confirming that the alcohol was oxidized within this period and allowing us to differentiate a period of active alcohol oxidation from a subsequent period of no alcohol oxidation.

It has been reported that women have a lower $\mathrm{ADH}$ activity in the gastric mucosa than men (Frezza et al. 1990), and that lower 'first-pass' gastric alcohol metabolism leads to higher blood alcohol levels. We had anticipated, therefore, that we would find higher blood alcohol levels in the women and that the alcohol disappearance curves of the men would lie below those of the women. Although this was the case the effect was small and not significant (Fig. 1). This may be due in part to the higher alcohol intakes prescribed in our study, equivalent to about $0.53 \mathrm{~g} / \mathrm{kg} \mathrm{BW}$ compared with $0.3 \mathrm{~g} / \mathrm{kg} \mathrm{BW}$ in the study of Frezza et al. (1990), leading to a greater proportion of the ingested alcohol reaching the portal circulation unmetabolized. The lack of any significant difference between men and women in their blood alcohol levels propagated through to their energy expenditure and substrate oxidation responses, which again showed no significant dependence on sex.

\section{Energy expenditure}

Both the male and female groups showed increased energy expenditure during the period of alcohol oxidation on the ADD protocol when compared with the CON and SUB protocols. The increase only accounted for $15 \%$ of the energy added; therefore, $85 \%$ must have been stored during this $6.5 \mathrm{~h}$ period. Dissipation of $10 \%$ of alcohol energy has been previously reported over a $4 \mathrm{~h}$ period after alcohol ingestion (Carpenter, 1940; Weststrate et al. 1990). Weststrate et al. (1990) observed that after $4 \mathrm{~h}$ thermogenesis was not complete in their studies, and in the first study of Rosenberg \& Durnin (1978) it was suggested that thermogenesis may be delayed when alcohol is taken with a meal. The measurements which we report here include and extend beyond the period of alcohol metabolism and so define the extent of alcohol-induced thermogenesis. Our value of $15 \%$ dissipation of alcohol energy is in close agreement with our previously reported value of $14 \%$ measured over $6 \mathrm{~h}$ in men (Sonko et al. 1994) and suggests a limit to alcohol-induced thermogenesis which is very similar to that associated with carbohydrate ingestion (Flatt, 1978). During the overnight period, after completion of alcohol oxidation, there was no significant excess thermogenesis in alcohol-treated subjects on either ADD or SUB protocols, so that over the whole period of observation $82.5 \%$ of the alcohol energy added was stored on the ADD protocol while on the SUB protocol subjects remained close to energy balance. These findings complement and extend previous experimental findings (Carpenter, 1940; Weststrate $e t$ al. 1990) and suggest that the dissipatory mechanisms proposed by Lieber $e t$ al. (1988) and by Lands \& Zakhari (1991) play no more than a minor role in alcohol disposal, at least at the representative levels of intake selected in our study. The absence of any significant thermogenesis on the SUB protocol contrasts with the observations of Suter et al. (1992) who found a $4 \%$ increase in $24 \mathrm{~h}$ energy expenditure when alcohol was 
substituted for $25 \%$ of dietary energy. However, it reinforces our view that alcohol provides energy with an efficiency similar to that of other energy substrates.

\section{Protein, carbohydrate and fat oxidation}

The priority given to the oxidation of ingested energy substrates is inversely related to the body's capacity to store them; thus alcohol, which cannot be stored and so must be detoxified by oxidation, may be expected to assume the highest priority for oxidation above protein, then carbohydrate and finally fat. As protein intake was not manipulated in this study and as its storage cannot be readily promoted, our prediction was that this study would not produce any changes in protein oxidation. This expectation was fulfilled; no significant differences in protein oxidation were found between any of the treatments. This contrasts, however, with the finding of decreased protein oxidation after intravenous infusion of alcohol reported by Shelmet et al. (1988). Alcohol is a potent suppressor of gluconeogenesis, which may offer an explanation for the suppression of protein oxidation in the Shelmet et al. (1988) subjects who were fasted overnight, in contrast to our subjects who had received breakfast and lunch before alcohol administration.

Carbohydrate oxidation was suppressed by $15 \%$ of the alcohol energy in the $6.5 \mathrm{~h}$ following addition of alcohol. This small suppression is broadly in line with the findings of Shelmet et al. (1988) and our expectations from our previous work (Sonko et al. 1994). There was a degree of compensation for this suppression overnight such that there was no overall effect of the addition of alcohol on carbohydrate oxidation, a finding in agreement with the reports of Suter et al. (1992).

Carbohydrate oxidation was significantly reduced during the period of alcohol oxidation when dietary carbohydrate was partly replaced by alcohol on the SUB protocol. The suppression amounted to $27 \%$ of the alcohol given, which was in turn equal in energy to the carbohydrate replaced. Some suppression of oxidation continued overnight, although this was not quite a significant effect. Over all, however, carbohydrate oxidation was significantly reduced by $40 \%$ of the substituted energy. Carbohydrate oxidation has been shown to adapt to changes in intake in an autoregulatory manner (Shetty et al. 1994) and, although there may be a small short-term effect of alcohol on carbohydrate oxidation, as suggested by the ADD results described previously, we view the reduction in carbohydrate oxidation on the SUB protocol as being primarily the adaptive response to a reduced carbohydrate intake rather than an alcohol-induced effect. If alcohol had continued to be substituted for carbohydrate in successive meals we would expect that ultimately carbohydrate oxidation would be reduced to match the reduced intake as stores assumed their new equilibrium.

The principal outcome of the present study is to be found in the fat oxidation results. During the period of alcohol oxidation, both on the SUB and the ADD protocols, the oxidation of fat was strongly and highly significantly suppressed. In the overnight period on the ADD protocol fat oxidation was similar to that of the CON protocol; however, the SUB protocol led to a small increase in fat oxidation which was almost significant $(P=$ 0.09 ) and complemented the reduced carbohydrate oxidation discussed previously. By the end of the $15.5 \mathrm{~h}$ experimental period, both groups showed a highly significant suppression of fat oxidation on both alcohol-treatment protocols. On the ADD protocol the greater part of the energy content of the alcohol $(74 \%)$ was stored as fat, an observation which would be supported by Shelmet et al. (1988), Weststrate et al. (1990), Suter et al. (1992), and by our own previous study (Sonko et al. 1994). On the SUB protocol the carbohydrate oxidation was not fully suppressed in proportion to the deficit in intake. Thus, relative to the CON condition, the SUB protocol resulted in a positive fat balance and negative carbohydrate balance; $59 \%$ of the alcohol energy was stored as fat. This contrasts with the 
outcome of our previous study (Sonko et al. 1994) in which manipulation occurred at lunch and in which suppressed carbohydrate and increased fat oxidation, together with an evening meal, subsequently restored substrate balance. In the current study the period following alcohol oxidation was one of sleep, during which the metabolic flexibility to reestablish substrate balance by transferring energy demands from carbohydrate to fat may be limited by the need to maintain a carbohydrate supply to the brain while other organs rest (McKenna et al. 1993). It appears from the current results that compensation for the effects of evening alcohol intake would have to be made within the context of the next day's food intake and energy substrate selections.

We conclude that energy ingested as alcohol is not simply dissipated as thermogenesis. Alcohol leads predominantly to the storage of an equivalent amount of fat when taken in addition to energy requirements. Any subsequent compensation for this must lie beyond the period of the present study. The consequences of replacement of dietary carbohydrate with alcohol seem to be dependant on subsequent diet and have yet to be fully elucidated. Our work has extended and reinforced previous laboratory-based studies but it has brought us little nearer to resolving the conflict between the results of laboratory studies and the survey results of workers such as Colditz et al. (1991). Moreover, it has not revealed any significant differences between men and women in their responses to alcohol which could shed light on the sex differences reported by Colditz et al. (1991). The study, however, does point us towards investigation of the response of the subjects and their substrate stores to the presentation of $a d l i b$. food on the days following the supplementation and substitution of dietary energy with alcohol.

The authors would like to record their thanks to all those who supported this work, and in particular Dr Marinos Elia for medical cover; Heather Tulett and Pauline Hordern, our night nurses; Elaine Collard and Judith Wills for preparation of the diets and of course our volunteer subjects who must remain anonymous!

\section{REFERENCES}

Badawy, A. A. B. (1978). The metabolism of alcohol. Clinics in Endocrinology and Metabolism 7, $247-271$.

Brown, D., Cole, T. J., Dauncey, M. J., Marrs, R. W. \& Murgatroyd, P. R. (1984). Analysis of gaseous exchange in open circuit indirect calorimetry. Medical and Biological Engineering and Computing 22, 333-338.

Carpenter, T. M. (1940). The metabolism of alcohol: a review. Quarterly Journal of Studies on Alcohol 1, $201-226$.

Colditz, G. A., Giovannucci, E., Rimm, E. B., Stampfer, M. J., Rosner, B., Speizer, F. E., Gordis, E. \& Willett,

W. C. (1991). Alcohol intake in relation to diet and obesity in women and men. American Journal of Clinical Nutrition 54, 49-55.

Flatt, J. P. (1978). The biochemistry of energy expenditure. Recent Advances in Obesity Research, pp. $211-228$. London: Newman Publishing.

Frezza, M., di Padova, C., Pozzato, G., Terpin, M., Baraona, E. \& Leiber, C. S. (1990). High blood alcohol levels in women. The role of decreased alcohol dehydrogenase activity and first-pass metabolism. New England Journal of Medicine 322, 95-99.

Lands, W. E. M. \& Zakhari, S. (1991). The case of the missing calories. American Journal of Clinical Nutrition 54, 47-48.

Leibel, R. L., Dufour, M., Hubbard, V. S. \& Lands, W. E. M. (1993). Alcohol and calories: a matter of balance. Alcohol 10, 429-434.

Lieber, C. S., Lasker, J. M., DeCarli, L. M., Saeli, J. \& Wojtowicz, T. (1988). Role of acetone, dietary fat and total energy intake in induction of hepatic microsomal ethanol oxidising system. Journal of Pharmacological Experiments and Theories 247, 791-795.

Livesey, G. \& Elia, M. (1988). Estimation of energy expenditure, net carbohydrate utilization, and fat oxidation and synthesis by indirect calorimetry: evaluation of errors with special reference to the detailed composition of fuels. American Journal of Clinical Nutrition 47, 608-628.

McKenna, A. P. M., Murgatroyd, P. R., Goldberg, G. R. \& Prentice, A. M. (1993). Low carbohydrate oxidation during rest : a possible mediator of weight loss in the profoundly inactive? Proceedings of the Nutrition Society $52,280 \mathrm{~A}$. 
Murgatroyd, P. R., Davies, H. L. \& Prentice, A. M. (1987). Intra-individual variability and measurement noise in estimates of energy expenditure by whole body indirect calorimetry. British Journal of Nutrition 58, 347-356.

Murgatroyd, P. R., Sonko, B. J., Wittekind, A., Goldberg, G. R., Ceesay, S. M. \& Prentice, A. M. (1993). Noninvasive techniques for assessing carbohydrate flux. I. Measurement of depletion by indirect calorimetry. Acta Physiologica Scandinavica 147, 91-98.

Rosenberg, K. \& Durnin, J. V. G. A. (1978). The effect of alcohol on resting metabolic rate. British Journal of Nutrition 40, 293-298.

Schofield, W. N., Schofield, C. \& James, W. P. T. (1985). Basal metabolic rate - review and prediction, together with an annotated bibliography of source material. Human Nutrition: Clinical Nutrition 39C, Suppl. 1.

Shelmet, J. J., Reichard, G. A., Skutches, C. L., Hoeldtke, R. D., Owen, O. E. \& Boden, G. (1988). Ethanol causes acute inhibition of carbohydrate, fat and protein oxidation and insulin resistance. Journal of Clinical Investigation 81, 1137-1145.

Shetty, P. S., Prentice, A. M., Goldberg, G. R., Murgatroyd, P. R., McKenna, A. P. M., Stubbs, R. J. \& Volschenk, P. A. (1994). Alterations in fuel selection and voluntary food intake in response to iso-energetic manipulation of glycogen stores in humans. American Journal of Clinical Nutrition 60, 534-543.

Smart, G. (1989). Is the postwar drinking binge ending? Cross-national trends in per capita alcohol consumption. British Journal of Addiction 84, 743-748.

Sonko, B. J., Prentice, A. M., Murgatroyd, P. R., Goldberg, G. R., van de Ven, M. L. H. M. \& Coward, W. A. (1994). Effect of alcohol on postmeal fat storage. American Journal of Clinical Nutrition 59, 619-625.

Sulkunen, P. (1976). Production, consumption and recent changes in consumption of alcoholic beverages. British Journal of Addiction 71, 115-122.

Suter, M. P., Schutz, Y. \& Jéquier, E. (1992). The effect of ethanol on fat storage in healthy subjects. New England Journal of Medicine 326, 983-987.

Weststrate, J. A., Wunnink, J., Duerenberg, P. \& Hautvast, J. G. A. J. (1990). Alcohol and its acute effects on resting metabolic rate and diet-induced thermogenesis. British Journal of Nutrition 64, 413-425. 